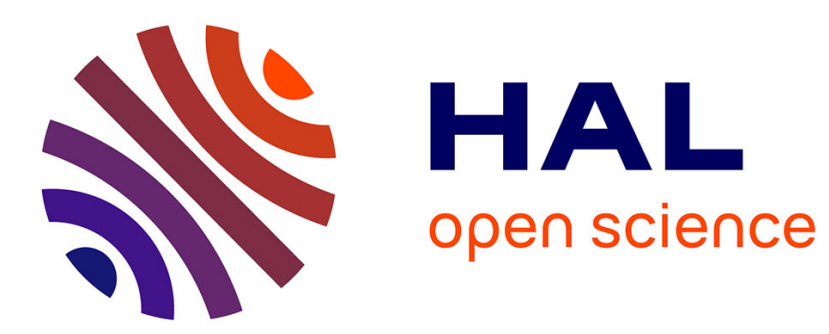

\title{
InKS, a Programming Model to Decouple Performance from Algorithm in HPC Codes
}

Ksander Ejjaaouani, Olivier Aumage, Julien Bigot, Michel Mehrenberger, Hitoshi Murai, Masahiro Nakao, Mitsuhisa Sato

\section{To cite this version:}

Ksander Ejjaaouani, Olivier Aumage, Julien Bigot, Michel Mehrenberger, Hitoshi Murai, et al.. InKS, a Programming Model to Decouple Performance from Algorithm in HPC Codes. Repara 2018 - 4th International Workshop on Reengineering for Parallelism in Heterogeneous Parallel Platforms, Aug 2018, Turin, Italy. pp.1-12, 10.1007/978-3-030-10549-5_59 . hal-01890132

\section{HAL Id: hal-01890132 \\ https://hal.science/hal-01890132}

Submitted on 8 Oct 2018

HAL is a multi-disciplinary open access archive for the deposit and dissemination of scientific research documents, whether they are published or not. The documents may come from teaching and research institutions in France or abroad, or from public or private research centers.
L'archive ouverte pluridisciplinaire HAL, est destinée au dépôt et à la diffusion de documents scientifiques de niveau recherche, publiés ou non, émanant des établissements d'enseignement et de recherche français ou étrangers, des laboratoires publics ou privés. 


\title{
InKS, a Programming Model to Decouple Performance from Algorithm in HPC Codes
}

\author{
Ksander Ejjaaouani ${ }^{1,2 凶}$, Olivier Aumage ${ }^{2,3}$, Julien Bigot ${ }^{1}$, Michel \\ Mehrenberger $^{4}$, Hitoshi Murai ${ }^{5}$, Masahiro Nakao ${ }^{5}$, and Mitsuhisa Sato ${ }^{5}$ \\ 1 Maison de la Simulation, CEA, CNRS, Univ. Paris-Sud, UVSQ, \\ Université Paris-Saclay, Gif-sur-Yvette, France julien.bigot@cea.fr \\ 2 Inria \{ksander.ejjaaouani, olivier. aumage\}@inria.fr \\ ${ }^{3}$ LaBRI, Bordeaux, France \\ 4 IRMA, Université de Strasbourg, Strasbourg, France mehrenbe@math.unistra.fr \\ ${ }^{5}$ Riken AICS, Kobe, Japan \{h-murai,masahiro.nakao,msato\}@riken.jp
}

\begin{abstract}
Existing programming models tend to tightly interleave algorithm and optimization in HPC simulation codes. This requires scientists to become experts in both the simulated domain and the optimization process and makes the code difficult to maintain and port to new architectures. This paper proposes the INKS programming model that decouples these two concerns with distinct languages for each. The simulation algorithm is expressed in the $\mathrm{INKS}_{\text {pia }}$ language with no concern for machine-specific optimizations. Optimizations are expressed using both a family of dedicated optimizations DSLs (INKSo) and plain $\mathrm{C}++$. INKSo relies on the INKS $_{\text {pia }}$ source to assist developers with common optimizations while $\mathrm{C}++$ is used for less common ones. Our evaluation demonstrates the soundness of the approach by using it on synthetic benchmarks and the Vlasov-Poisson equation. It shows that INKS offers separation of concerns at no performance cost.
\end{abstract}

Keywords: programming model $\cdot$ separation of concerns $\cdot$ HPC $\cdot$ DSL

\section{Introduction}

It is more and more common to identify simulation as the third pillar of science together with theory and experimentation. Parallel computers provide the computing power required by the more demanding of these simulations. The complexity and heterogeneity of these architectures do however force scientists to write complex code (using vectorization, parallelization, accelerator specific languages, etc.) These optimizations heavily depend on the target machine and the whole code has to be adapted whenever it is ported to a new architecture.

As a result, scientists have to become experts in the art of computer optimizations in addition to their own domain of expertise. It is very difficult in practice to maintain a code targeting multiple distinct architectures. One fundamental cause for this situation is the tight interleaving of two distinct concerns imposed by most programming models. On the one hand, the algorithm comes 
from the expertise of the domain scientist and does not depend on the target architecture. On the other hand, optimization is the expertise of optimization specialists and has to be adapted for every new architecture.

Many approaches have been proposed to improve this situation in the form of libraries or languages. Approaches based on automated optimization processes typically isolate the algorithmic aspects very well but restrict their domain of applicability and/or the range of supported optimizations. Approaches based on optimization tools and libraries enable optimization specialists to express common optimizations very efficiently but leave others mixed with the algorithm.

In this paper, we propose the Independent Kernel Scheduling (INKS) programming model to separate algorithm from optimization choices in HPC simulation codes. We define the $\mathrm{INKS}_{\text {pia }}$ language used to express the algorithm of an application independently of its optimization. Such a program can be optimized with the INKS $\mathrm{O}$ family of domain-specific languages (DSLs) for common optimizations or $\mathrm{C}++$ for less common optimizations.

This paper makes the following contributions: 1) it defines the INKS programming model and its platform-independent algorithmic language INKS $\mathrm{Iia}_{\text {pia }} ; \mathbf{2}$ ) it proposes an implementation of INKS with two optimization DSLs, INKSO/Loop and $\mathrm{INKS}_{\mathrm{O} / \mathrm{XMP}}$; and 3 ) it evaluates the approach on the synthetic NAS parallel benchmarks [3] and on the $6 \mathrm{D}$ Vlasov-Poisson solving with a semi-Lagrangian method.

The remaining of the paper is organized as follows. Section 2 presents and analyzes related work. Section 3 describes the INKS programming model and its implementation. Section 4 presents the $6 \mathrm{D}$ Vlasov-Poisson problem and its implementation using InKS while Section 5 evaluates the approach. Finally, Section6 concludes the paper and identifies some perspectives.

\section{Related works}

Many approaches are used to implement optimized scientific simulations. A first widely used approach is based on imperative languages such as Fortran, C or $\mathrm{C}++$. Libraries like MPI extend this to distributed memory with message passing. Abstractions very close to the actual execution machine make fine-tuning possible to achieve good performance on any specific architecture. It does however require encoding complex optimizations directly in the code. As there is no language support to separate the algorithm and architecture-specific optimizations, tedious efforts have to be applied [10] to support performance portability. Algorithm and optimizations are instead often tightly bound together in codes.

A second approach is thus offered by tools (libraries, frameworks or language extensions) that encode classical optimizations. OpenMP [5] or Kokkos [4] support common shared-memory parallelization patterns. E.g, Kokkos offers multidimensional arrays and iterators for which efficient memory mappings and iteration orders are selected independently. E.g, UPC [8] or XMP [14] support the partitioned global address space paradigm. In XMP, directives describe array distribution and communications between nodes. These tools offer gains of pro- 
ductivity when the optimization patterns they offer fit the requirements. The separation of optimizations from the main code base also eases porting between architectures. Even if expressed more compactly optimizations do however remain mixed with the algorithm and only cover part of the requirements.

A third approach pushes this further with tools that automate the optimization process. For example, PaRSEC [1] or StarPU [1] support the many-tasks paradigm. In StarPU, the user expresses its code as a DAG of tasks with data dependencies that is automatically scheduled at runtime depending on the available resources. Another examples are SkeTo [18] or Lift [16] that offer algorithmic skeletons. Lift offers a limited set of parallel patterns whose combinations are automatically transformed by an optimizing compiler. Automating optimization improves productivity and clearly separate these optimizations which improves portability. The tools do however not cover the whole range of potential optimizations such as the choice of work granularity inside tasks. The algorithm remains largely interleaved with optimization choices even with this approach.

A last approach is based on DSLs that restrict optimizations, such as Pochoir [17] or PATUS [6], DSLs for stencil problems. In Pochoir, the user only specifies a stencil (computation kernel and access pattern), boundary conditions and a space-time domain while all optimizations are handled by a compiler. DSLs restrict the developer to the expression of the algorithm only, while optimizations are handled independently. This ensures a very good separation of these aspects. The narrower the target domain is, the more efficient domain and architecturespecific optimizations are possible. However, it makes it less likely for the tool to cover the needs of a whole application. Real-world applications can fall at the frontier between the domains of distinct DSLs or not be covered by a single one. Performance then depends on the choice of DSLs to use and the best choice depends on the target architecture leading to new portability issues.

To summarize, one can consider a continuum of approaches from very general approaches where the optimization process is manual to more and more domain specific where the optimization process can be automated. The more general approaches support a large range of optimizations and application domains but yield high implementation costs and low separation of concerns and portability. The more automated approaches reduce implementation costs and offer good separation of concerns and portability but restrain the range of supported domains and optimizations. Ideally, one would like to combine all these advantages: 1) the domain generality of imperative languages, 2) the ease of optimization offered by dedicated tools and 3) the separation of concerns and performance portability offered by DSLs. The following section describes the INKS programming model that aims to combine these approaches to offer such a solution.

\section{The InKS programming model}

This section describes the core of our contribution, the INKS programming model. This approach is based on the use of distinct languages to express algo- 
rithm and optimization choices; thus enforcing their separation. The algorithm of the simulation consists in the set of values computed, the formula used to produce each of them as well as the simulation inputs and outputs. We include in optimization choices all that is not the algorithm, such as the choice of a computing unit for each computation, their ordering, the choice of a location in memory for each value, etc. Multiple optimization choices can differ in performance but simulation results depend on the algorithm only.

The INKS approach is summarized in Figure1. The INKS $_{\text {pia }}$ language is used to express the algorithm with no concern for optimization choices. A compiler generates non-optimized, generic choices automatically from this specification for test purposes. The $\mathrm{INKS}_{\mathrm{O}}$ family of DSLs is used to define common optimizations efficiently while $\mathrm{C}++$ is used to describe arbitrarily complex optimizations. Many versions of the optimization choices can be devised to optimize for multiple targets.

The remaining of the section describes INKS $\mathrm{S}_{\text {pia }}$ and proposes two preliminary $\mathrm{INKS}_{\mathrm{O}}$ DSLs. INKS $\mathrm{O} / \mathrm{XMP}$ handles domain decomposition and inter-node communications while $\mathrm{INKS}_{\mathrm{O} / \text { Loop }}$ focuses on efficient loops.

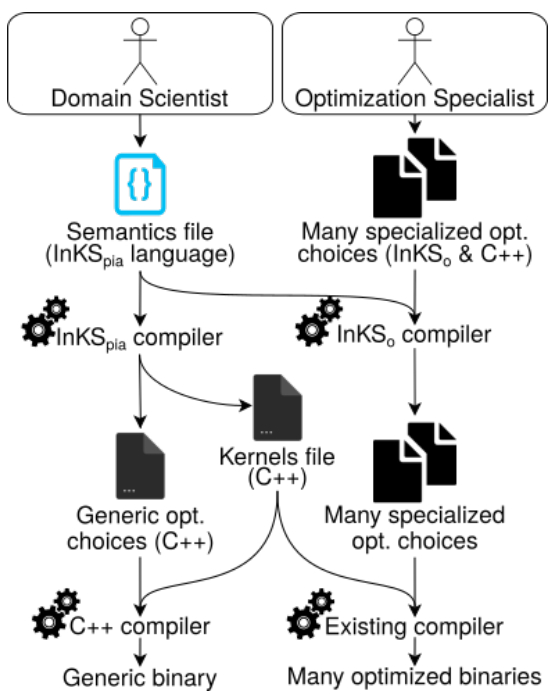

Fig. 1: The INKS model

The INKS $_{\text {pia }}$ language. In INKS pia 2 (illustrated in Listing 1), values are stored in infinite multidimensional arrays based on dynamic single assignment (DSA, each coordinate can only be written once). Memory placement of each coordinate is left unspecified. Computations are specified by kernel procedures that 1) take as parameters data arrays and integer coordinates; 2) specify the coordinate they might read and will write in each array; and 3) define either a $\mathrm{C}++$ or INKS implementation. An INKS implementation defines kernels $v a$ lidity domains: coordinates where $\mathrm{C}++$ kernels can generate values in arrays. Kernel execution order is left unspecified. The simulation entry point is a kernel marked public. This specifies a parameterized task graph (PTG) [7]. This representation covers a large range of problems but imposes a few limitations. Mostly, all the problem parameters must be known at launch time, it does for example not support adaptive mesh or time-steps. It is still possible to express these concerns outside INKS pia and call the INKS implementation multiple times with different parameters.

The $\mathrm{INKS}_{\text {pia }}$ compiler 2] extracts $\mathrm{C}++$ functions from $\mathrm{INKS}_{\text {pia }}$ kernels and generates a function with correct but non-optimized loops and memory allocations to execute them. Arbitrarily complex versions of these can also be written 


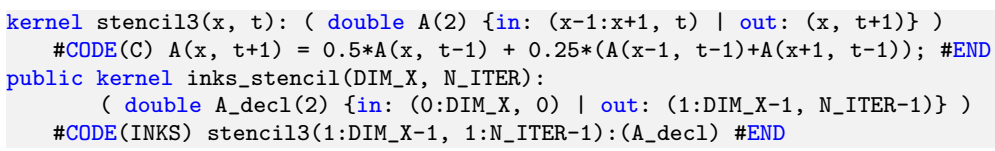

Listing 1: 1D stencil computation on a $2 \mathrm{D}$ domain in $\mathrm{INKS}_{\text {pia }}$

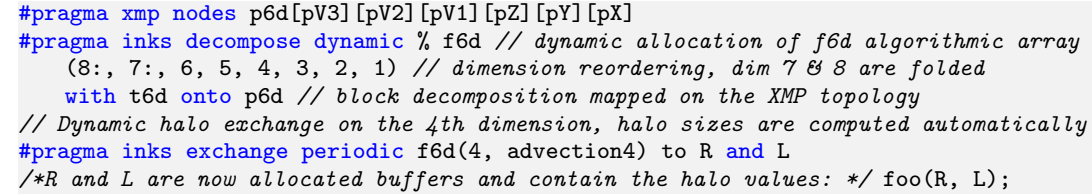

Listing 2: $6 \mathrm{D}$ decomposition and dynamic halo exchange in $\mathrm{INKS}_{\mathrm{O} / \mathrm{XMP}}$

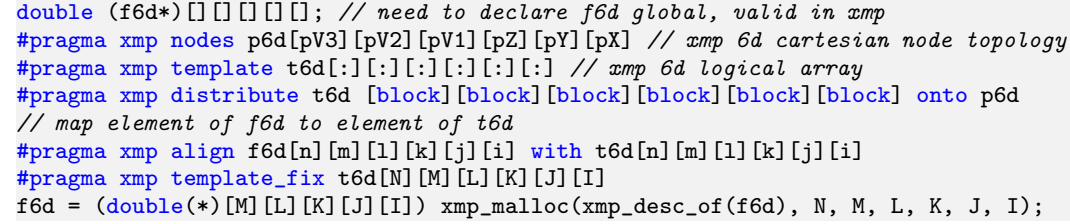

Listing 3: XMP code generated for the f6D decomposition of Listing2

manually in plain $\mathrm{C}++$ and rely on existing optimization tools. These functions can be called from any existing code whose language supports the $\mathrm{C}$ calling convention. However, that approach requires information present in $\mathrm{INKS}_{\text {pia }}$ to be repeated. The INKSo DSLs thus interface optimization tools with INKS pia.

The $\mathrm{INKS}_{\mathrm{O} / \mathrm{XMP}}$ optimization language. INKS $\mathrm{O} / \mathrm{XMP}$ (illustrated in Listing 2 ) handles distributed memory domain decomposition by combining $\mathrm{C}$ and directives based on XMP and adapted for INKS. The compiler replaces these directives by $\mathrm{C}$ and XMP code (Listing 3 ). The inks decompose directive supports static or dynamic allocation of arrays from the algorithm. The domain size is extracted from INKS $\mathrm{S}_{\text {pia }}$ source and the user only has to specify its mapping onto memory. As in XMP, INKS $\mathrm{O} / \mathrm{XMP}$ supports domain decomposition mapped onto an XMP topology. In addition, it supports dimension reordering and folding which consists in reusing the same memory address for subsequent indices in a given dimension. This feature is important to reuse memory due to the DSA nature of INKS pia arrays. The exchange directive supports halo exchanges. The required halo size is automatically extracted from the algorithm and the user only has to specify when to execute the communications and in what dimension. While XMP requires halo values to be stored contiguously with the domain, INKS $_{\mathrm{O} / \mathrm{XMP}}$ support a dynamic halo extension where halo values are stored in dedicated, dynamically allocated buffers to reduce memory footprint.

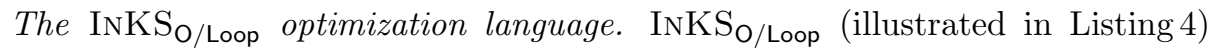
offers to specify manually loop nests for which the compiler generates plain 


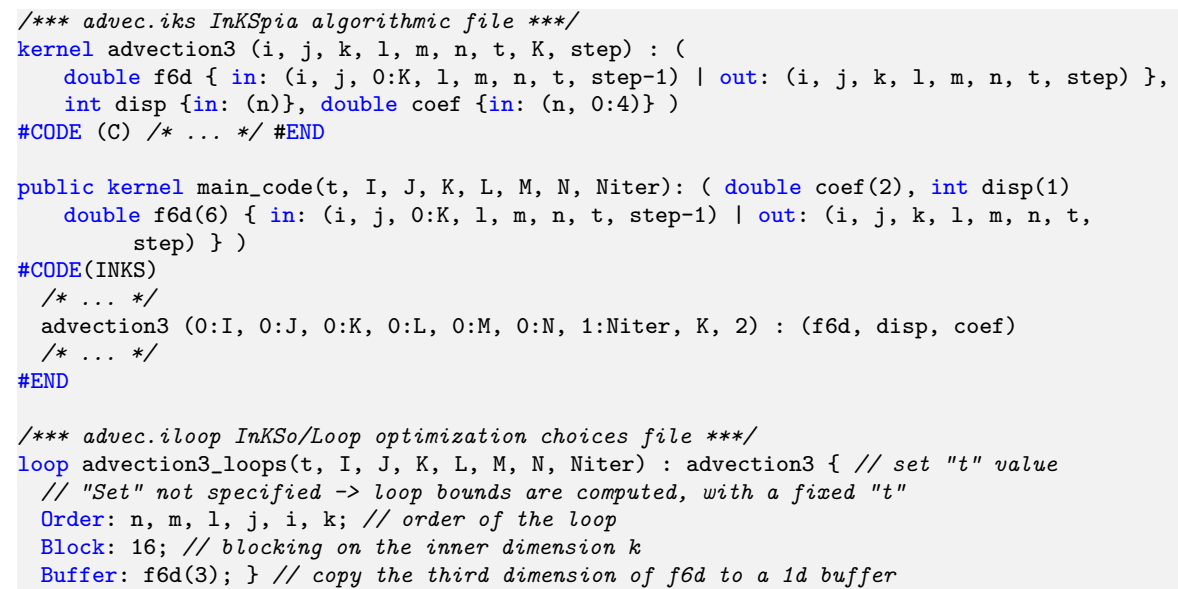

Listing 4: A loop nest in $\operatorname{INKS}_{\text {pia }}$ optimized in $\mathrm{INKS}_{\mathrm{O} / \text { Loop }}$

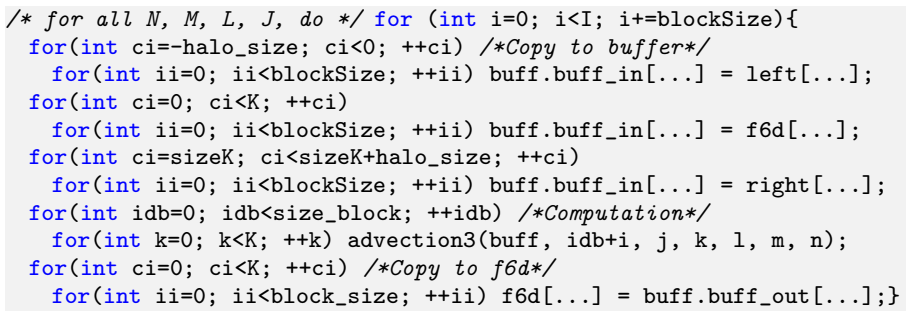

Listing 5: $\mathrm{C}++$ code generated for the loop nest of Listing 4

$\mathrm{C}++$ loops (Listing 5). Plain $\mathrm{C}++$ is usable with $\mathrm{InKS}_{\mathrm{O} / \text { Loop. The loop keyword }}$ introduces a nest optimization with a name, the list of parameters from the algorithm on which the loop bounds depend and a reference to the optimized kernel. Loop bounds can be automatically extracted from INKS $S_{\text {pia }}$, but the Set keyword makes it possible to restrict these bounds. The Order keyword specifies the iteration order on the dimensions and the Block keyword enables the user to implement blocking. It takes as parameters the size of block for the loops starting from the innermost one. If there are less block sizes than loops, the remaining loops are not blocked. The Buffer keyword supports copying data in a local buffer before computation and back after to ensure data continuity and improve vectorization. The compiler uses data dependencies from the algorithm to check the validity of the loops order and generate vectorization directives where possible.

\section{The 6D Vlasov/Poisson problem}

The 6D Vlasov-Poisson equation, presented in (1), describes the movement of particles in a plasma and the resulting electric field. We study its resolution for 
a single species on a $6 \mathrm{D}$ Cartesian mesh with periodic boundary conditions. We solve the Poisson part using a fast Fourier transform (FFT) and rely on a Strang splitting (order 2 in time) for the Vlasov part. This leads to $61 \mathrm{D}$ advections: 3 in the space dimensions $\left(x_{1}, x_{2}, x_{3}\right)$ and 3 in the velocity dimensions $\left(v_{1}, v_{2}, v_{3}\right)$. Each 1D advection relies on a Lagrange interpolation of degree 4 . In the space dimensions, we use a semi-Lagrangian approach where the stencil is not applied around the destination point but at the foot of characteristics, a coordinate known at runtime only. This is described in more details in [15.

$$
\left\{\begin{array}{l}
\frac{\partial f(t, x, v)}{\partial t}+v \cdot \nabla_{x} f(t, x, v)-E(t, x) \cdot \nabla_{v} f(t, x, v)=0 \\
-\Delta \phi(t, x)=1-\rho(t, x) \\
E(t, x)=-\nabla \phi(t, x) \\
\rho(t, x)=\int f(t, x, v) d v
\end{array}\right.
$$

The main unknown is $f$ ( $\mathrm{f} 6 \mathrm{D}$ in the code), the distribution function of particles in $6 \mathrm{D}$ phase space. Due to the Strang splitting, a first half time-step of advections is required after $\mathrm{f} 6 \mathrm{D}$ initialization but before the main time-loop. These advections need the electric field $E$ as input. $E$ is obtained through the FFT-based Poisson solver that in turn needs the charge density $\rho$ as input. $\rho$ is computed by a reduction of $\mathrm{f} 6 \mathrm{D}$. The main time-loop is composed of 3 steps: advections in space dimensions, computation of the charge density (reduction) and electric field (Poisson solver) and advections in velocity dimensions.

The $6 \mathrm{D}$ nature of $\mathrm{f} 6 \mathrm{D}$ requires a lot of memory, but the regularity of the problem means it can be distributed in blocks with good load-balancing. Halos are required to hold values of neighbors for the advections. Connected halo zones would increase the number of points in all dimensions and consume too much memory. Split advections mean that halos are required in a single dimension at a time though. We therefore use dynamic halos composed of two buffers, one for each boundary of the advected dimension (denoted "right" and "left") as shown on Figure 2 Listing 2 corresponds to the INKS $\mathrm{S}_{\mathrm{O}} \mathrm{XMP}$ implementation of this strategy.

Advections are the main computational cost of the problem, accounting for $90 \%$ of the sequential execution time. Six loops surround the stencil computation of each advection and in a naive version, the use of a modulo to handle periodicity and application along non-contiguous dimensions slow down the computation. To enable vectorization and improve cache use, we copy f6D elements into contiguous buffers along with the left and right halos. Advections are applied on these buffers before copying them back into f6D. Blocking further improves performance by copying 16 elements at a time. Listing 5 corresponds to the INKS $\mathrm{O}_{\mathrm{O} \text { Loop }}$ implementation of these optimizations. 


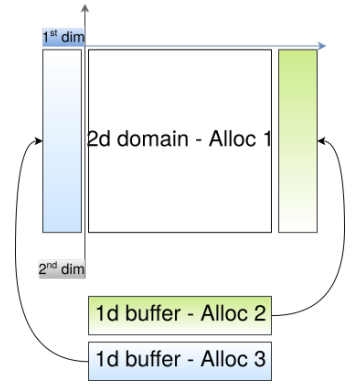

(a) Buffers used as halo regions in the first dimension

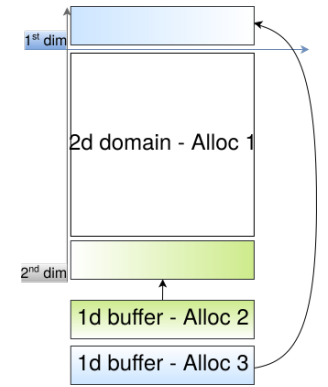

(b) Buffers used as halo regions in the second dimension

Fig. 2: Dynamic halo exchange representation on a 2D domain

\section{Evaluation}

This section evaluates the INKS model on the NAS parallel benchmark, a simple stencil code and the $6 \mathrm{D}$ Vlasov-Poisson problem. Plain $\mathrm{C}++$ is used for the synthetic benchmarks optimization while $\mathrm{INKS}_{\mathrm{O} / \mathrm{XMP}}$, INKS $\mathrm{I}_{\mathrm{O} / \text { Loop }}$, plain $\mathrm{C}++$ and $\mathrm{C}$ with XMP are used for Vlasov-Poisson. All codes are compiled with Intel 18 compiler (-03 -xHost), Intel MPI 5.0.1 and executed on the Poincare cluster (Idris, France) with 32 GB RAM and two Sandy Bridge E5-2670 CPUs per node and a QLogic QDR InfiniBand interconnect.

\subsection{Synthetic benchmarks}

We have implemented 4 out of the 5 sequential NAS benchmark kernels (IS, FT, $\mathrm{EP}$ and $\mathrm{MG}$ ) in $\mathrm{INKS}_{\text {pia }}$ and optimized them with plain $\mathrm{C}++$. The $\mathrm{C}++$ version 9 , is used as reference. We have also implemented a $3 \mathrm{D}$ heat equation resolution by finite differences (7-point stencil) with two distinct $\mathrm{C}++$ optimization strategies from a single $\mathrm{INKS}_{\text {pia }}$ source. Both strategies comes from the reference [12. One uses double buffering (Heat/Buf) and the other implements a cache oblivious strategy (Heat/Obl).

The NAS CG kernel relies on indirections not expressible in the PTG model of INKS $_{\text {pia. }}$. Its implementation would thus have to rely on a large $\mathrm{C}++$ kernel whose optimization would be mixed with the algorithm. INKS $\mathrm{Sia}_{\text {can }}$ however be used to express all other NAS kernels as well as the 3D heat equation solver. Even if not as expressive as $\mathrm{C}$ or Fortran, INKS $\mathrm{S}_{\text {pia }}$ covers the needs of a wide range of simulation domains and offers abstractions close to the execution machine rather than from a specific simulation domain. Among others, it can express computations such as FFTs or stencils with input coordinates unknown at compile-time.

INKS separates the specification of algorithm and optimization in distinct files. Multiple optimization strategies can be implemented for a single algorithm, 
InKS, a Programming Model to Decouple Performance \& Algorithm in HPC

\begin{tabular}{rccccccc} 
& \multicolumn{2}{c}{ Execution time (second) } & & \multicolumn{2}{c}{ Complexity } \\
\cline { 2 - 4 } Benchmark & Reference & InKS & Rel. dev. & & Ref. & InKS \\
\hline NAS/FT & $62.43( \pm 0.57)$ & $62.86( \pm 0.71)$ & $0.68 \%$ & & 6 & 5 \\
NAS/IS & $3.39( \pm 0.00)$ & $3.44( \pm 0.00)$ & $1.47 \%$ & & 55 & 52 \\
NAS/MG & $5.10( \pm 0.02)$ & $4.73( \pm 0.06)$ & $-7.25 \%$ & & 20 & 12 \\
NAS/EP & $76.43( \pm 0.21)$ & $76.47( \pm 0.22)$ & $0.05 \%$ & & 19 & 19 \\
Heat/Buf & $3.05( \pm 0.01)$ & $2.97( \pm 0.06)$ & $-2.58 \%$ & & 5 & 3 \\
Heat/Obl & $2.43( \pm 0.01)$ & $2.05( \pm 0.02)$ & $-15.59 \%$ & & 22 & 13
\end{tabular}

Table 1: Execution time of the $\mathrm{C}++$ and INKS implementations of the sequential NAS benchmark, class B - Time/iteration of the 3D heat equation (7point stencil), size $\left(1024^{3}\right)$ - Median and standard deviation of 10 executions - GNU Complexity score of the implementation.

as shown for the 3D heat equation where each relies on a specific memory layout and scheduling. It thus offers a clear separation of algorithm and optimization.

Finding the right metric to evaluate the easiness of writing a code is a difficult question. As illustrated in Listing 4 however, algorithm expression in $\operatorname{INKS}_{\text {pia }}$ is close to the most naive $\mathrm{C}$ implementation where loops are replaced by INKS validity domains with no worry for optimization. The specification of optimization choices is close to their expression in $\mathrm{C}++$. Table 1 compares the GNU complexity score of INKS optimizations to the reference code. INKS scores are slightly better because kernels extracted from the algorithm hide computations and thus, part of the complexity. In addition, the use of $\mathrm{C}++$ to write optimizations let optimization specialists reuse their preexisting knowledge of this language. These considerations should not hide the fact that some information has to be specified both in the $\mathrm{INKS}_{\text {pia }}$ and $\mathrm{C}++$ files with this approach leading to more code overall.

Regarding performance, the INKS approach makes it possible to express optimizations that do not change the algorithm. Optimizations of the four NAS parallel benchmarks and 3D heat equation solver in INKS were trivial to implement and their performance match or improve upon the reference as presented in Table1. Investigation have shown that Intel ICC 18 does not vectorize properly the reference versions of Heat/Obl and NAS/MG. The use of the Intel ivdep directive as done on the INKS versions leads to slightly better performance.

\subsection{Vlasov-Poisson}

We evaluate $\mathrm{INKS}_{\mathrm{O} / \mathrm{XMP}}$ and $\mathrm{INKS}_{\mathrm{O} / \text { Loop }}$ on Vlasov-Poisson separately as they target different optimizations and are not usable together currently. A first experiment focuses on the sequential aspects with the intra-node optimization of the $v_{1}$ advection using either $\mathrm{INKS}_{\mathrm{O} / \text { Loop }}$ or plain $\mathrm{C}++$. A second experiment focuses on the parallel aspects with the charge density computation, the Poisson

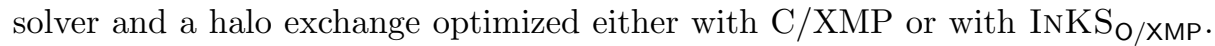
The reference is the Fortran/MPI implementation from the Selalib library [13]. 


\begin{tabular}{rccc} 
Version & time/advection & GFLOP/s & \% peak core perf. \\
\hline Selalib (Fortran) & $4.81 \mathrm{~s}$ & 1.12 & $5.36 \%$ \\
INKS (C++) & $3.76 \mathrm{~s}$ & 1.43 & $6.87 \%$ \\
INKS (INKS O/Loop) & $3.61 \mathrm{~s}$ & 1.49 & $7.16 \%$
\end{tabular}

Table 2: Comparison between Fortran, $\mathrm{C}++$ and $\mathrm{INKS}_{\mathrm{O} / \text { Loop }}$ version of the $v_{1}$ advection on a $32^{6}$ grid (double precision) on a single E5-2670 core with vectorization. Median of 12 executions

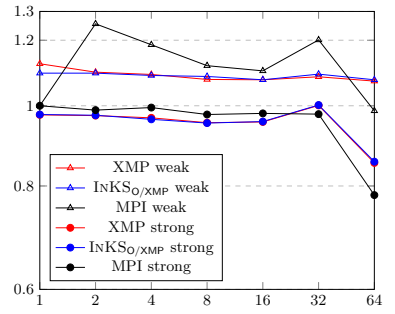

(a) f6D reduction

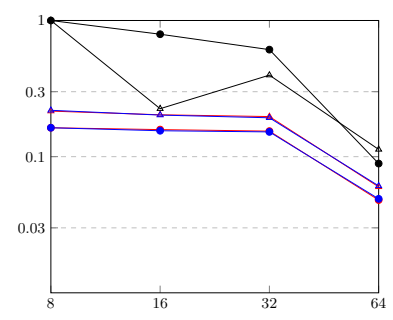

(b) Poisson solver

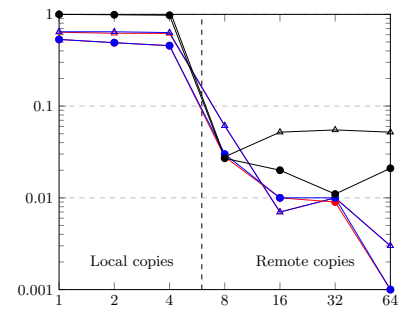

(c) Dynamic halo exchanges

Fig. 3: Weak and strong scaling for 3 parts of the Vlasov-Poisson solver up to 64 nodes ( 1 process/node) on a $32^{6}$ grid divided among processes (strong scaling) or $16^{6}$ grid per process (weak scaling). Median of 10 executions.

Distinct files for both concerns in INKS makes possible to write a unique $\mathrm{INKS}_{\text {pia }}$ algorithm and multiple versions of optimization choices. Four optimization choices are implemented based on one $\mathrm{INKS}_{\text {pia }}$ source : (1) INKS $\mathrm{O} / \mathrm{XMP}$, (2) $\mathrm{C}$ with XMP, (3) InKS $\mathrm{X}$ /Loop and (4) plain $\mathrm{C}++$. This proves, to some extent, that the separation of concerns is respected. As of now, INKS $\mathrm{O} / \mathrm{XMP}$ and $\mathrm{INKS}_{\mathrm{O} / \text { Loop }}$ are not usable together since $\mathrm{INKS}_{\mathrm{O} / \text { Loop }}$ relies on $\mathrm{C}++$ that $\mathrm{XMP}$ does not support. We plan to address this limitation in the future.

For the $v_{1}$ advection, both the $\mathrm{C}++$ and $\mathrm{INKS}_{\mathrm{O} / \text { Loop }}$ optimizations of the INKS code achieve performance similar to the reference as shown in Table2, For the parallel aspects, the INKS $\mathrm{S}_{\mathrm{XMP}}$ optimization offers performance similar to $\mathrm{XMP}$ as shown on Figure 3. The performance is comparable to MPI on the reduction operation but MPI is faster on the Poisson solver and the halo exchanges. At the moment, it seems that XMP does not optimize local copies which slows down the Poisson solver. Besides, XMP directives used for the halo exchanges are based on MPI RMA which make the comparison with MPI Send/Receive complex. Still, MPI is much harder to program: more than 350 lines of MPI and Fortran are required to handle domain decomposition, remapping for FFT and halo exchange in Selalib vs. 50 lines in XMP and 15 in INKS $_{\mathrm{O} / \mathrm{XMP}}$.

The INKSo family of DSLs enables the developer to specify optimization choices only while algorithmic information is extracted from $\mathrm{INKS}_{\text {pia }}$ code. This is illustrated by Listings 2 presenting the $\mathrm{INKS}_{\mathrm{O} / \mathrm{XMP}} 6 \mathrm{D}$ domain decomposition, 
and 3 presenting the XMP version. Both are equivalent, but the $\mathrm{INKS}_{\mathrm{O} / \mathrm{XMP}}$ expects only optimization choices parameters. Hence, one can test another memory layout, such as a different dimension ordering, by changing only a few parameters, while multiple directives must be modified in XMP. Similarly, with $\mathrm{INKS}_{\mathrm{O} / \text { Loop }}$ (Listing/4, developers can easily test different optimization choices that would be tedious in plain $\mathrm{C}++$. Since $\mathrm{INKS}_{\mathrm{O} / \mathrm{XMP}}$ and $\mathrm{INKS}_{\mathrm{O} / \text { Loop }}$ are respectively usable with $\mathrm{C}$ and $\mathrm{C}++$, INKS does not restrict the expressible optimization choices: one can still implement optimizations not handled by INKSo in $\mathrm{C} / \mathrm{C}++$. Moreover, operations such as halo size computation or vectorization capabilities detection are automatized using the algorithm. In summary, the approach enables optimization specialists to focus on their specialty which make the development easier.

\section{Conclusion and future works}

In this paper, we have presented the INKS programming model to separate algorithm $\left(\operatorname{INKS}_{\text {pia }}\right)$ and optimization choices (INKSo \& $\mathrm{C}++$ ) and its implementation supporting two DSLs : INKS $\mathrm{O}_{\text {/Loop }}$ for loop optimizations and INKS $\mathrm{O} / \mathrm{XMP}$ for domain decomposition. We have evaluated INKS on synthetic benchmarks and on the Vlasov-Poisson solving. We have demonstrated its generality and its advantages in terms of separation of concerns to improve maintainability and portability while offering performance on par with existing approaches.

While this paper demonstrates the interest of the INKS approach, it still requires some work to further develop it. We plan to apply the INKS model on a range of different problems. We will improve the optimization DSLs; base INKS $_{\mathrm{O} / \text { Loop }}$ on existing loop optimization tools and ensure good interactions with $\mathrm{INKS}_{\mathrm{O} / \mathrm{XMP}}$. We also want to target different architectures to demonstrate the portability gains of the INKS approach.

\section{References}

1. Augonnet, C., Thibault, S., Namyst, R., Wacrenier, P.A.: Starpu: A unified platform for task scheduling on heterogeneous multicore architectures. Concurr. Comput. : Pract. Exper. 23(2), 187-198 (Feb 2011). https://doi.org/10.1002/cpe.1631

2. Aumage, O., Bigot, J., Ejjaaouani, K., Mehrenberger, M.: Inks, a programming model to decouple performance from semantics in simulation codes. Tech. rep. (2017), https://hal-cea.archives-ouvertes.fr/cea-01493075

3. Bailey, D.H., Barszcz, E., Barton, J.T., Browning, D.S., Carter, R.L., Dagum, L., Fatoohi, R.A., Frederickson, P.O., Lasinski, T.A., Schreiber, R.S., et al.: The nas parallel benchmarks. The International Journal of Supercomputing Applications 5(3), 63-73 (1991)

4. Carter Edwards, H., Trott, C.R., Sunderland, D.: Kokkos. J. Parallel Distrib. Comput. 74(12), 3202-3216 (Dec 2014). https://doi.org/10.1016/j.jpdc.2014.07.003

5. Chandra, R., Dagum, L., Kohr, D., Maydan, D., McDonald, J., Menon, R.: Parallel Programming in OpenMP. Morgan Kaufmann Publishers Inc., San Francisco, CA, USA (2001) 
6. Christen, M., Schenk, O., Burkhart, H.: PATUS: A Code Generation and Autotuning Framework for Parallel Iterative Stencil Computations on Modern Microarchitectures. In: Parallel \& Distributed Processing Symposium (IPDPS), 2011 IEEE International. pp. 676-687. IEEE (May 2011). https://doi.org/10.1109/ipdps.2011.70

7. Cosnard, M., Jeannot, E.: Compact dag representation and its dynamic scheduling. Journal of Parallel and Distributed Computing 58(3), 487 514 (1999). https://doi.org/10.1006/jpdc.1999.1566, http://www.sciencedirect. com/science/article/pii/S0743731599915666

8. El-Ghazawi, T., Carlson, W., Sterling, T., Yelick, K.: UPC: Distributed Shared Memory Programming (Wiley Series on Parallel and Distributed Computing). Wiley-Interscience (2005)

9. Griebler, D., Lff, J., Fernandes, L., Mencagli, G., Danelutto, M.: Efficient nas benchmark kernels with c++ parallel programming (01 2018)

10. Höhnerbach, M., Ismail, A.E., Bientinesi, P.: The vectorization of the tersoff multibody potential: An exercise in performance portability. In: Proceedings of the International Conference for High Performance Computing, Networking, Storage and Analysis. pp. 7:1-7:13. SC '16, IEEE Press, Piscataway, NJ, USA (2016), http://dl.acm.org/citation.cfm?id=3014904.3014914

11. Hoque, R., Herault, T., Bosilca, G., Dongarra, J.: Dynamic task discovery in parsec: A data-flow task-based runtime. In: Proceedings of the 8th Workshop on Latest Advances in Scalable Algorithms for Large-Scale Systems. pp. 6:1-6:8. ScalA '17, ACM, New York, NY, USA (2017). https://doi.org/10.1145/3148226.3148233

12. Kamil, S.: Stencilprobe: A microbenchmark for stencil applications (2012), http:// people.csail.mit.edu/skamil/projects/stencilprobe/, accessed: 2017-08-25

13. Kormann, K., Reuter, K., Rampp, M., Sonnendrcker, E.: Massively parallel semilagrangian solution of the $6 \mathrm{~d}$ vlasov-poisson problem. (Oct 2016)

14. Lee, J., Sato, M.: Implementation and performance evaluation of xcalablemp: A parallel programming language for distributed memory systems. In: 2010 39th International Conference on Parallel Processing Workshops. pp. 413-420 (Sept 2010). https://doi.org/10.1109/ICPPW.2010.62

15. Mehrenberger, M., Steiner, C., Marradi, L., Crouseilles, N., Sonnendrücker, E., Afeyan, B.: Vlasov on gpu (vog project)******. ESAIM: Proc. 43, 37-58 (2013). https://doi.org/10.1051/proc/201343003

16. Steuwer, M., Remmelg, T., Dubach, C.: Lift: A functional data-parallel ir for high-performance gpu code generation. In: 2017 IEEE/ACM International Symposium on Code Generation and Optimization (CGO). pp. 74-85 (Feb 2017). https://doi.org/10.1109/CGO.2017.7863730

17. Tang, Y., Chowdhury, R.A., Kuszmaul, B.C., Luk, C.K., Leiserson, C.E.: The pochoir stencil compiler. In: Proceedings of the Twenty-third Annual ACM Symposium on Parallelism in Algorithms and Architectures. pp. 117-128. SPAA '11, ACM, New York, NY, USA (2011). https://doi.org/10.1145/1989493.1989508

18. Tanno, H., Iwasaki, H.: Parallel skeletons for variable-length lists in sketo skeleton library. In: Proceedings of the 15th International Euro-Par Conference on Parallel Processing. pp. 666-677. Euro-Par '09, Springer-Verlag, Berlin, Heidelberg (2009). https://doi.org/10.1007/978-3-642-03869-3_63 